\title{
A Design of Direct Current Supply System for X-ray Flaw Detector Controller
}

\author{
Yuguo Yue \\ School of Liaoning Mechatronics College, Liaoning 51000, China \\ yueyuguo@126.com
}

Keywords: DC power, DC inverter, DC voltage compensation, X-ray generator, DC controller, DC electric fan, portable, $\mathrm{X}$-ray flaw detector, nondestructive testing.

\begin{abstract}
Based on the existing X-ray flaw detector, the writer tried to design a direct current supply system for X-ray flaw detector controller so that a portable DC X-ray flaw detector can be made in China to fit the need of the professional market.

As one of the regular NDT technologies, X-ray detection technique has played a really important role in our professional field, which can be classified into two species- portable or mobile. Nowadays the portable X-ray flaw detectors are used mostly because the X-ray detection are mostly be done on engineering sites.

When the X-ray flaw detector work on outdoor engineering sites like long-distance pipeline or tank detection, as there aren't any power supplies, we always use a electric generator to support the flaw detector, however as detecting work piece places always change, the electric generator need to be moved frequently, which increases labor intensity, decreases work efficiency and consume a large quantity of gasoline, makes a lot of noise and pollutes the environment. Besides, some construction places are inside steep mountains with few narrow meandering footpaths, which increase difficulties of the construction. It becomes a big problem that the electric generators need to meet the power supply requirements of X-ray flaw detectors on construction sites, which will affect detecting constructions. If we still use the normal field inspection method: double-wall single-image radiography with X-ray flaw detector powered by oil fired generator, the quantity of detecting workers and the cost of detecting will increase rapidly. It is difficult to move the electric generator at steep district and the rate of detecting progress will be so slow that the construction wouldn't be finished as schedules. Because of the thin wall pipe, using $\gamma$-ray can't meet the standard for the basic requirement of blackness and sensitivity, meanwhile, the requirement of safety will be stricter if the $\gamma$-ray is used. As a result, using the direct current supply system for X-ray flaw detector is a good solution which will solve the problem of X-ray flaw detector in the steep slope zone of the power supply and detection problem.
\end{abstract}

\section{DC X-ray flaw detector}

A DC portable X-ray detector is mainly composed of X-ray generator, cooling system, DC controller, power supply and outside charging device, and it is a kind of portable X-ray generator, which can be carried by 2 to 4 people on roads that are unable to open to traffic such as mountain roads or farmlands, as a result, it can replace the ordinary X-ray flaw detector. A schematic diagram of DC X-ray flaw detector is shown as below. 


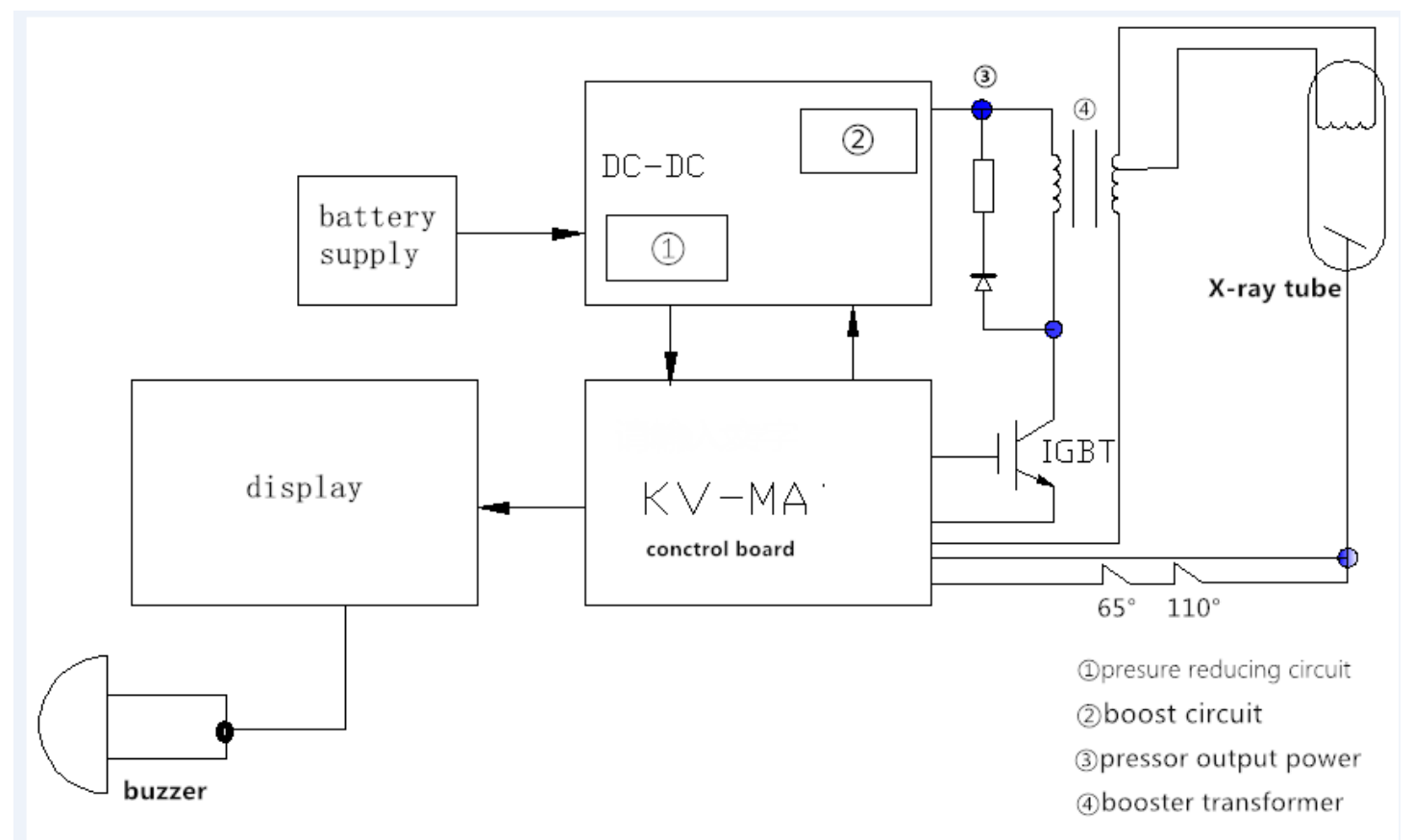

Fig. 1 DC X-ray flaw detector schematic diagram

The writer take measures about the weight and the reliability of the console, for example, the console of previous X-ray flaw detector often use a semi-automatic exposure parameter preselection device, some with waveform conversion device, which has a complex circuit. Now the complex circuit and the large parts are replaced by integrated components, which will reduce the weight and the volume of the console, so that it will generally be only 10 kilogram. To improve the reliability of the console, the writer will optimize his design and control and measure with microcomputer system, which greatly improve the reliability and accuracy. As we can see, the design and manufacture of the DC portable X-ray flaw detector is one of the domestic hot spot of nondestructive testing equipment at present.

It is still in the discussion stage in our country about the development of the DC portable X-ray flaw detector and there is no application and product sold. Nowadays, an imported DC portable $\mathrm{X}$-ray flaw detector is very expensive and there is no spot, with at least 3 months ordering period, besides, technical consultation and after service is difficult to guarantee. Combining with the development situation and application status, exploiting DC portable X-ray flaw detector is a good solution for the big problem of the demand of it.

\section{A design of DC power supply system}

The console of X-ray flaw detectors is a device to give power to the machine and control it, whose (circuit) part use DC-DC module, MCU and IGBT. DC-DC module regulated power supply has compact size, exceptional performance and it is easy to use. And MCU has many advantages such as small volume, mature circuit and high reliability. High voltage pulse circuit use IGBT( insulated gate bipolar transistor) to reduce loss to the greatest extent so routing needs to be simplified so that it's easy to maintain and strong electricity parts need to be put nearby to separate strong electricity and weak electricity in case of the disturbance of control system. At last, because of high requirement of fabrication process, components need to be selected grimly.

The power supply is DC high voltage power supply and considering the requirements of the console about convenience, time and adjustment, the writer chooses to use DC inverter with a 120V DC power supply( a battery with fission installation to reduce monomer weight and easy to carry) and voltage-compensated circuit. Besides, to provide adequate protection for alternate detection, the writer prefers to use a set of 15Ah battery with $100 \%$ sufficient electricity( with charging device), which can continuous work for more than 3 hours( $80 \%$ discharge capacity) based on $240 \mathrm{kv}$ per minute and 10 ampere. Furthermore, based on the design of power supply-console-ray machine, the 
writer tries to make it that it has convenient connection, flexible operation, and shall not affect the transportation.

As the conversion efficiency of the X-ray flaw detector is very low, every X-ray flaw detector should equip a good cooling system, which use $24 \mathrm{~V}$ DC brushless electric fan so that it can continuous work for more than 10000 hours and its speed can be controlled.

\section{Summary}

The developed electric supply is applied on many domestic DC portable X-ray flaw detector, which is welcomed by many nondestructive testing companies and meet the needs of the industry market. Practice has proved, the DC portable X-ray flaw detector has many advantages as below.

3.1 A independent power supply is only $14 \mathrm{Kg}$, as heavy as $1 / 6$ of a generator, so it's easy to be carried, which effectively solves the problem that when the X-ray flaw detector is used in a steep slope location, the generator can't be moved to the detection place so that the detection can't be carried out because there is no way to go, and it saves a lot of valuable time that is wasted by carrying the generator, as a result, manpower and material resources are reduced, so as the construction risk.

3.2 It is suitable for $10 \sim 400 \mathrm{~mm}$ pipe, which meet the requirement of the radiographic inspection by double-wall double-image or double-wall single-image in steep slope section such as Oil \& gas productivity construction.

3.3When exposure conditions and darkroom disposal conditions are the same, using the DC $\mathrm{X}$-ray flaw detector to transilluminate X-ray photographic plate can make its blackness higher than the plate transilluminated by ordinary X-ray flaw detector, and its technical performance index is stable, which meet the SY/T4109-2005 standard requirement.

3.4Transillumination large diameter pipeline such as $\Phi 219 \times 7 \mathrm{~mm}$ pipeline butt welds can short exposure time so that detecting efficiency can be improved.

3.5Compared with imported products, it has a obvious price advantage that only equivalent to $1 / 20$ of the imported products, what's more, it has many other advantages such as daily maintenance, easy maintenance, repair parts guaranteed. All of these realize the goal that use domestic products to replace imported products, and it saves a large amount of foreign exchange.

\section{References}

[1] Yuguo Yue, Radiographic Testing, China Machine Press, Beijing, 2013.

[2] SY/T4109-2005 Nondestructive testing of oil and gas steel pipeline radiographic testing. 